\title{
Pre-touch Proxemics: Moving the Design Space of Touch Targets from Still Graphics towards Proxemic Behaviors
}

\author{
Ilhan Aslan \\ Augsburg University \\ Germany \\ aslan@hcm-lab.de
}

\author{
Elisabeth André \\ Augsburg University \\ Germany \\ andre@hcm-lab.de
}

\begin{abstract}
Proxemic touch targets continuously change in relation to a user's hand in mid-air before a physical touch occurs. Previous work has, for example shown that expanding targets are capable to improve target acquisition performance on touch interfaces. However, it is unclear how proxemic touch targets influence user experience (UX) in a broader sense, including hedonic qualities. Towards closing this research gap the paper reports on two user studies. The first study is a qualitative study with five experts, providing in-depth insights on a variety of change-types (e.g., size, form, color) and how they, for example, influence perceived functional and aesthetic qualities of proxemic touch targets. A follow-up user study with 36 participants explores the UX of a proxemic touch target compared to non-proximal versions of the same target. The results highlight a positive significant effect of the proxemic design on both pragmatic and hedonic qualities.
\end{abstract}

\section{CCS CONCEPTS}

- Human-centered computing $\rightarrow$ Interaction techniques;

\section{KEYWORDS}

Touch, mid-air input, proxemics, user experience, design

\section{ACM Reference Format:}

Ilhan Aslan and Elisabeth André. 2017. Pre-touch Proxemics: Moving the Design Space of Touch Targets from Still Graphics towards Proxemic Behaviors. In Proceedings of 19th ACM International Conference on Multimodal Interaction (ICMI'17). ACM, New York, NY, USA, 9 pages. https: //doi.org/10.1145/3136755.3136808

\section{INTRODUCTION}

In this paper, we address the ongoing efforts towards the design and implementation of dynamic touch interfaces, which are capable to sense fingers in 3D space proximal to the touch screen and single touch targets on the screen (e.g.,[1, 2, 7, 17, 29]). These efforts make use of the expanded interaction space above the screen in order to improve the usability of touch interfaces either by improving target acquisition performance or by creating novel short cuts and

This is the author's version of the work. It is posted here for your personal use. Not for redistribution. The definitive Version of Record was published in:

ICMI'17, November 13-17, 2017, Glasgow, UK

(C) 2017 Association for Computing Machinery.

ACM ISBN 978-1-4503-5543-8

https://doi.org/10.1145/3136755.3136808 reducing the complexity of screen-based touch interfaces. For example, Aslan et al. [1] have proposed to improve the usability of touch interfaces in cars by expanding touch targets in relation to the drivers approaching fingers. In order to reduce the complexity of mobile touch interfaces, Hinckley et al. [17] have, for example, proposed to adapt touch interfaces to postures of an approaching hand, allowing the interface to recognize user intentions, such as an intention to tap with the thumb or to zoom with two fingers. The aim of this paper is to contribute to the existing body of related work by contrasting previous research on usability of dynamic touch interfaces with research in user experience (UX) design in a broader sense, including hedonic qualities of touch target designs. To keep it simple, we will refer hereafter to dynamic touch targets that continuously adapt their appearance in relation to a proximal hand/finger as "proxemic" touch targets.

Our main assumption is that, there is a general quality in proxemic touch targets beyond the outcome of user interaction (e.g., task completion time, error rate), which is often the focus of "traditional" usability studies. We believe that by employing continuous and relational interaction, proxemic touch targets can significantly reinforce their (physical and "emotional") relation/connection to users and thus their perceived experiential qualities during interaction. In this paper we address two research questions: (i) what are the perceived differences when different features of touch targets are changed, and (ii) do proxemic target designs have a good UX (i.e., are they perceived as attractive and desirable)?

In order to address the research questions, two user studies were conducted. The first study explored with five experts perceived experiential qualities of proxemic touch targets when only one feature at a time of a set of appearance features (i.e., size, position, color, form/shape, orientation, semantic) is dynamically changed. A second study with 36 participants compared the effect of proxemic touch targets with two control groups (i.e., static graphics and nonproxemic animation), applying the attrakDiff ${ }^{1}$ questionnaire as an analytical tool to measure perceived UX. The results support our initial assumptions by confirming a positive significant impact of the proxemic touch target design on perceived pragmatic quality and perceived hedonic qualities.

In the following section, we first present background and related work in touch target acquisition and proxemic interaction. Then both user studies are described in depth, including the prototypes and methods employed. After presenting the results in detail, we conclude by discussing future design implications for touch interfaces with proxemic touch targets, which include a potential shift from designing static graphics towards designing proxemic behaviors.

\footnotetext{
${ }^{1} \mathrm{http}: / /$ attrakdiff.de/index-en.html
} 
a)

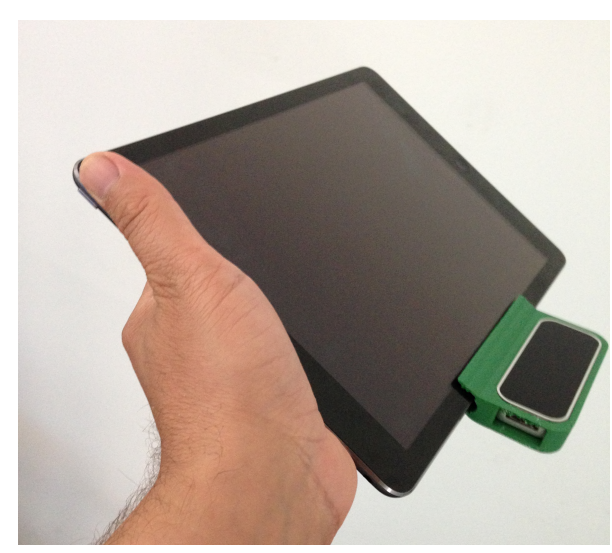

b)

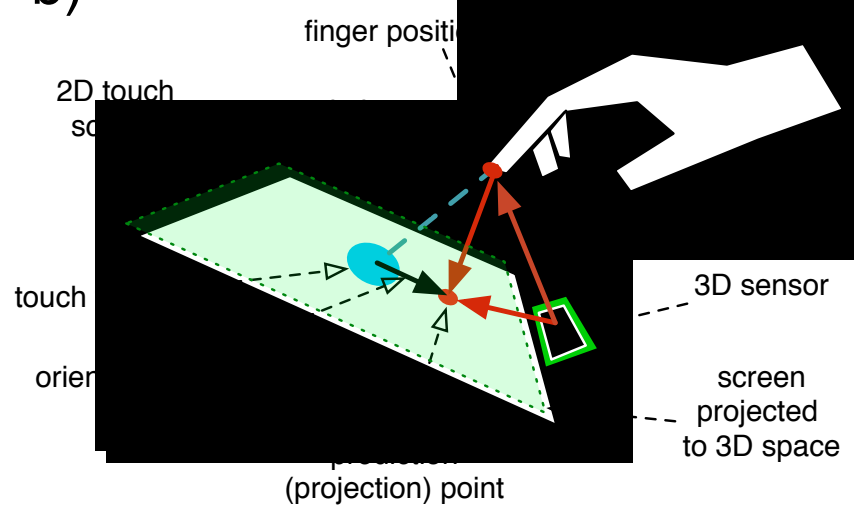

Figure 1: a) Overview of the prototype, including the 3D printed cover for the Leap Motion sensor b) Abstract presentation of the interaction setup with the measures highlighted, which are used to adapt touch targets dynamically for proxemic interaction.

\section{BACKGROUND}

Screen-based touch interfaces are the dominant form of interaction on most of today's mobile devices. The directness of touch makes use of human sensory-motoric skills, which humans have improved over their lifespan, interacting through touch with their physical environment, including carrying toys around as children or making love as adults. Thus, touch-based interaction feels more natural and requires less time learning, compared to other pointing based human-computer interaction techniques, such as a computer mouse. As humans, we understand our physical environment through making sense of the space around us and movement within this space [27]. Many of the objects we touch in our everyday lives are non-animated and provide no reactions until we touch them and perceive a touch sensation in our fingers.

However, we share the space we live in with other human and non-human agents, such as animals, which are capable to sense us back and understand our spatial behavior and respond through movement (i.e., spatial feedback).

How humans interpret and make sense of spatial feedback during interpersonal interaction have been studied in the social sciences, providing a theoretical foundation (i.e., Proxemics [14]), which has the potential to enrich human-computer interaction, since similar to touch humans make sense of spatial feedback and apply spatial behavior for non-verbal communication in everyday situations. For example, when a person A orients themselves towards a person B, A signals B interest and an intention to make contact. B may face $\mathrm{A}$ in return and interact with $\mathrm{A}$ or turn away to signal disinterest.

\subsection{Proxemic, Relational, and Organic Interfaces}

The non-verbal interactivity between two bodies which is governed by measures of body-proximity and body-orientation has been of special interest for the design of proxemic human-computer interfaces. Inspired by Edwards Hall's Proxemics theory [14] Proxemic
Interactions (e.g., [12]) has been proposed in the field of HumanComputer Interaction (HCI) as a general stance towards the design of ubiquitous computing (ubicomp) applications. Ballendat et al. [5] have, for example, proposed proxemic interaction to mediate simultaneous interactions of multiple users or to utilize directed attention to other people or objects in a co-shared multi-body space. Thus, Proxemic Interactions in HCI has been associated mainly with spatial information between separate physical bodies, such as devices and non-digital objects co-sharing a (typically room-sized) physical space, or players [22] in a game context.

It is important that proxemic interaction is not considered constrained to absolute positions of two bodies in space and their orientation towards each other but there is quality in how bodies react in relation to their changing proximity and orientation. For example, Hemmert et al. [16] have studied how users' perceive a mobile phone if the body of the mobile phone is capable to move in response to an approaching hand. Similarly, Pederson et al. [24] have studied how shape changing hand-held devices are perceived based on a large scale study using videos.

Common to the exemplary observations made by Hemmert et al. [16] and Pederson et al. [24] is that animating objects (either by changing the posture or shape of the physical body) in relation to a user's approaching hand results in users associating the feedback with nature and living agents, such as animals. Parkes et al. [23] have argued more generally that movement is associated with "being alive" and thus a key ingredient of a category of interfaces they refer to as organic kinesthetic interfaces (KOIs).

While Parkes et al. [23] argue that KOIs are a category of interfaces which are capable to move in response to a user, they put emphasis on the quality of physical movement (including tactile feedback and movement sound) in comparison to motion on screens, which essentially is virtual. However, screen-based interfaces are highly flexible, providing the capacity to adapt to changing requirements due to, for example, mobility or constraints associated with small sized screens. 


\subsection{Proxemic Touch Targets}

Within the last five years the body of related work in proxemic touch targets has been increasing rapidly with previous work, studying expanding and/or moving touch targets, which aim to improve target selection and acquisition performance for different situations, such as touch target acquisition in cars [1], in collaboration settings [2], and for selecting a small-sized target from a group of targets [29]. Related work in target acquisition on touch screens often refers to Fitts's law [9], which is relevant whenever aimed movements, such as pointing is part of the user input. Fitts's law provides a model for aimed movements, describing a trade-off between speed and accuracy [21], which is based on the (physical) measures distance of an aimer to a target and size of the target. Both of these measures of touch targets can be adapted in relation to an approaching hand in order to improve speed and accuracy of user input (e.g., [1, 2, 7, 20, 29]).

In addition to improving touch target acquisition in diverse situations, others have suggested to address typical issues with mobile interaction, such as small sized screens, one-handed usage, and occlusion. Chen et al. [7], for example, suggested to combine pretouch or post-touch gestures with touch allowing alternative forms to perform operations, such as to zoom by tabbing the screen followed by circling with the finger above the screen. Hinckley et al. suggested to adapt touch interfaces depending on the posture of the approaching hand [17], for example to differentiate a two-fingerzoom intention from thumb-tap intention on the screen and thus reduce information overload on the screen.

In summary, many have identified the experiential qualities of physical movement as feedback, which the following quote by Parkes et al. [23] highlights.

... human beings possess a deeply rooted response to motion, recognizing innately in it a quality of "being alive" provoking a significantly deeper and emotional response from users. [23]

While there is related work in exploring experiential qualities of, for example, shape-changing and moving physical artifacts (e.g., $[11,24,26,28])$ there is a lack of similar studies exploring touch targets on screens.

However, previous work by Aslan et al. [1,2] suggest otherwise, indicating that even when touch targets only change their size or position in relation to an approaching finger they already inspire a feeling of connectedness [1] and associate agency [2] with touch targets on the screen (e.g., users assume that touch targets have the intention to help them to tap them when targets expand in response to an approaching finger). Setting up on their initial results, we aim to understand and explore the relationship between a user and a touch target during an aimed movement, when the touch target is capable to sense the users' hand mid-air and react to it.

\section{PROTOTYPE}

\subsection{Hardware Setup and Software}

In order to turn traditional touch targets into proxemic touch targets we combined a tablet device (i.e., an iPad air) with a Leap Motion controller (see Figure 1a), using a 3D printed cover. The cover fixes the Leap Motion's position to the iPad screen, allowing the software to work precisely after calibration even if the device is being moved afterwards. Previous work has established the Leap
Motion sensor's precision [13] and the sensor has been applied to precision sensitive applications, such as the design of mid-air authentication gestures [3]. In our implementation the user needs to perform a preliminary calibration task, which serves as the basis for mapping and synchronizing 2D screen positions with mid-air finger positions in 3D. During the calibration task three targets are presented at different corners of the touch screen. When the user touches targets, each target memorizes its position in 3D and expands thereafter depending on the user's finger proximity to the center of the touch target (FPCT).

The target expansions provide user feedback and once the user is satisfied with the expansion behavior of all three touch targets the calibration can be finished. The three 3D positions are used to project a 2D plane, which corresponds to the physical iPad screen position relative to the Leap Motion sensor. Using vector projection the mid-air 3D (index) finger position of the user is projected on the 2D screen of the iPad. We refer to this position on the iPad screen as the prediction point (PP). Furthermore, an orientation vector $(\mathrm{OV})$ from the center of the target to the PP is computed and used to calculate the orientation of the users' index finger to the target. The details of the measures used to synchronize $2 \mathrm{~d}$ touch input with 3D mid-air positions is illustrated in Figure $1 \mathrm{~b}$.

\subsection{Change-Types}

Rasmussen et al. [25] have provided a review of the design space of shape-changing interfaces, including a topology for the type of changes (e.g., orientation, form, volume), which we use as foundation. We have implemented a variety of change-types as proxemic touch target behaviors based on changing their size, position, form, orientation, color, or semantics. Figure 3 provides an overview of the different change-types, which we consider as probes to explore the first research question: exploring the perceived differences when different features of touch targets are changed. In the following we provide additional information on how change-types were implemented using FPCT, PP, and OV:

(1) Form-change: The form-change target is built from an area spanned by two bezier-curves, which change their form and consequently the area spanned by them in relation to an approaching finger. The bezier-curves change randomly (within a predefined space) and considering FPCT and OV. The result can look, for example like a "butterfly" or "mouth" when both curves change (by chance) in synchrony.

(2) Size-change: In the size-change implementation a target's initial size (100px) expands depending solely on FPCT (within the range up to $4 \mathrm{~cm})$ to a maximum size of $(200 \mathrm{px})$ in a linear fashion.

(3) Color-change: In this implementation the color is set randomly in the beginning. Depending on the FPCT the saturation of the color is adapted in a linear fashion with the highest level of saturation being achieved when the distance to the FPCT is less than $2 \mathrm{~cm}$.

(4) Position-change: In the position-change implementation the target moves towards the PP, but only if the PP's distance is less then 200px.

(5) Semantic-change: Depending on FPCT the target thumbnail image (see Figure 2) transitions from one randomly chosen 

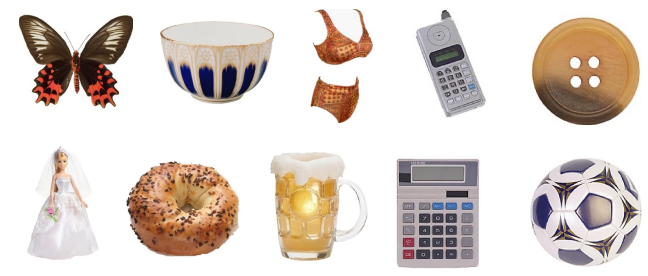

Figure 2: Overview of the images used for the semanticchange implementation.

image to an other image. We chose a diverse set of semantically different objects [19], including diverse perceived textures.

(6) Orientation-change: In this implementation a (round) target rotates (depending on $\mathrm{OV}$ ) towards the approaching finger of a user.

The size-change and position-change implementations are similar to the implementations in previous work by Aslan et al. $[1,2]$ who have explored proxemic touch targets to improve target acquision performance. As proposed by Gaver et al. [10] we used ambiguity as part of our probes (especially in form-change and semantic-change) to foster engagement and to allow users to interpret designs more personally. We did this to explore an otherwise very large design space spanned by the form-change and semantic change implementations.

\section{EXPLORATIVE USER STUDY WITH EXPERTS}

Five participants ( $\mathrm{m}$, between 25-33 years of age) with expertise in human-computer interaction (from our own lab who were not involved in the research) were invited to explore the target behaviors in individual sessions with a facilitator. We choose to use experts with a focus on interaction design with diverse specialization (e.g., UX design or game design) instead of novice users, since experts have the required skills and experiences to "read" early prototypes and understand associated ideas, while most novice users require more elaborated designs. The study consisted of two phases, an exploration phase and a reflection phase. In the beginning, the facilitator provided a brief overview of the prototype and introduced the exploration phase, asking the experts to try out each target behavior by themselves. The prototype offered a menu from which participants could switch between touch target behaviors and experience each change-type separately

In the exploration phase the experts could take as much time as they needed with each target behavior and compare target behaviors. The facilitator asked the experts to follow the procedure of (i) describing the target they interacted with, (ii) describing the target's behavior, (iii) interpreting the intention of the target as far as possible, and (iv) describing how the interaction felt. By using the "think aloud" technique [8] the experts were asked to verbally articulate their experiences when interacting with the prototypes. In the reflection phase the experts were asked to reflect why they preferred one behavior over an other and how reactive behaviors were different from the static version.

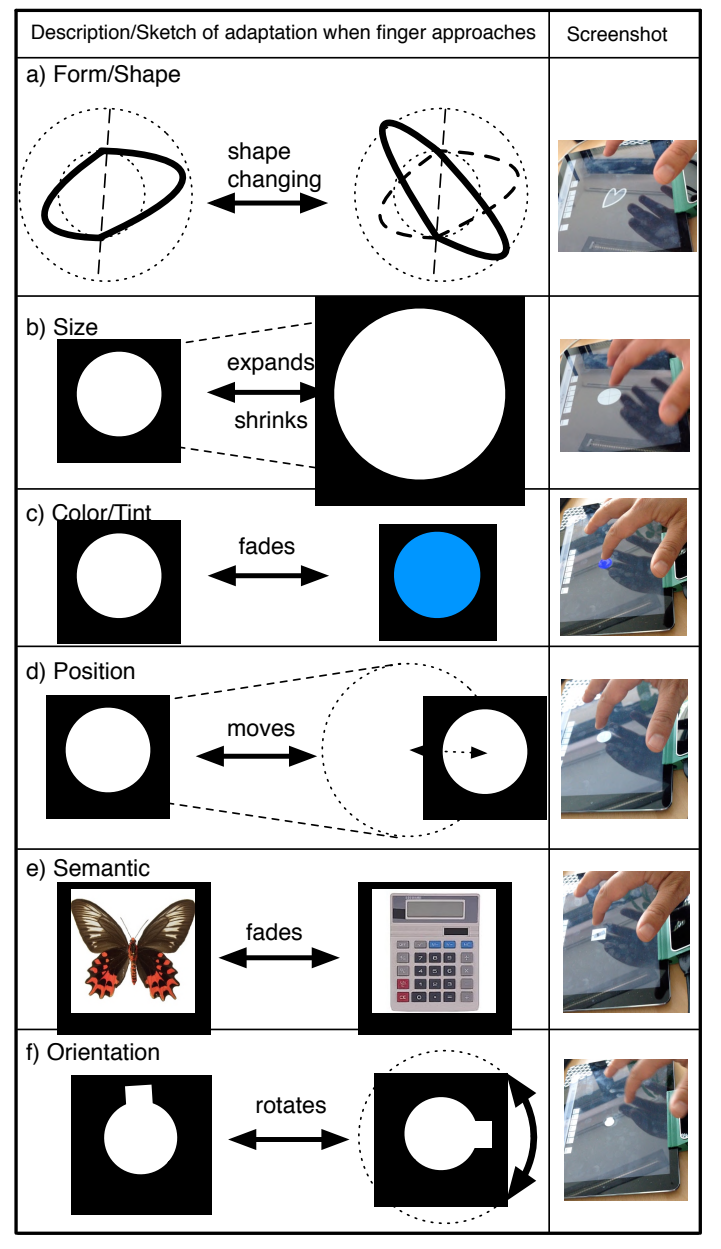

Figure 3: Screenshot of each implementation, demonstrating the variety of proxemic touch target behavior provided by the prototype.

All sessions were recorded and analyzed post-hoc, according to the basics of thematic analysis [6]. This qualitative methodology is typically used to identify, analyze, and report themes (patterns) within data. Sessions with experts took between 20 and 40 minutes. In the following we summarize the results focusing on three distinctive themes.

\subsection{Results and Discussion}

In all change-types, targets seem to communicate that they can be touched and that they are interactive. When describing the target object's behavior and their possible intentions, experts used terms that are typically used to describe behavior of living, animated things, such as "it is tenacious" or it wants to be squeezed". One expert stated during the form-change implementation: "my first impressions was that it is a flesh eating plant". What could be observed is that over time experts referred to touch targets as if they were talking about intelligent (social) agents, such as "he is trying to help me push him". An interesting reaction, which we observed 
was when the experts were asked to try out the static version in the reflection phase. All experts stated that it felt weird (now). They argued that they must have gotten used to targets' somehow reacting to their aimed movements. One expert argued that it felt boring (now), another one stated: "this is dull" and "he is dead now". We observed that experts were disappointed that the target did not register aimed movement and, thus, their interest, which concluded in some "irritation" in the experts. In the following we summarize the three themes that emerged with respect to previous work and our own theoretical position: (i) feedback and feedforward perception, (ii) peripheral and implicit interaction, and (iii) aesthetic vs. functional experiences.

Experts described targets' behaviors as feedback or feedforward actions, referring to functions and interactions (e.g., gestures) targets may support. Especially, the target that oriented itself towards an approaching hand was perceived as communicating that it can and wants to be turned like a volume knob (e.g., to increase or decrease volume in applications). Two of the experts explicitly used the term affordances and how the proxemic target behavior was experienced as a way for the targets to communicate their affordances (e.g., being a separate touchable element on the screen).

Considering the second theme peripheral and implicit interaction, comments were made about how proximity information could be used to integrate screens more seamlessly into the surrounding environment by adapting color schemes and semantics depending on if users directly interact with touch interfaces or not. It was suggested that especially in multi-user settings, proxemic targets could transition from a view for direct interaction into a view more suitable for multiple observers, integrating these observers into collaborative interactions. Another expert, argued that he preferred the color-change implementation most because it could be used to transition an interface during non-use into something ambient, which would not feel distracting or annoying in periphery.

The third theme aesthetic vs functional experiences highlights a contrast between two groups of change-types. One group of implementations was referred to as functional (i.e, clearly supporting users in their aiming tasks), the experience with the other group of implementations (i.e., form-change, color-change, semantic-change) was referred to as aesthetic. Overall, only one expert preferred the size-change implementation and one expert the orientation-change implementation the rest of the experts preferred either the colorchange or form-change implementation most. For example, one participant stated that he liked the form-change implementation and the color-change implementation best of all implementations, but he could not explain in detail why, other than stating that it was aesthetic, and it felt interesting and engaging.

In summary, we observed similar to the experiential qualities associated with physical movement when it is provided as feedback [23] that proxemic touch targets provoke emotional responses from the experts. Such responses are typically associated with interactions with other agents and an innate quality of "being alive". Thus, it seems that despite movement in proxemic touch targets being essentially virtual, there is still a potential to enhance the user experience compared to non-proxemic touch targets. Furthermore, we believe that the exploitation of the combined benefits of different change-types bears potential towards the creation of aesthetic and functional experiences.

\section{A PROXEMIC TOUCH TARGET DESIGN}

The results of the first explorative user study encouraged us to formulate the idea that combining multiple change-types (e.g., color-change and orientation-change) may improve in addition to functional qualities (e.g., make it feel easier to acquire targets) and aesthetic qualities (e.g., improve the overall attractiveness) of proxemic touch targets. In order to test this idea we implemented a design instance of a proxemic touch target (see Figure 4), which implements and combines the change-types: color-change, sizechange, form-change, and orientation-change. In Figure $4 \mathrm{a}$ we also illustrate key design decisions towards getting the design right. For the first design we considered to use only two ellipses with the first ellipse on a fixed position and the second ellipse capable to move towards an approaching finger of the user. Inspired by related work (e.g., [25]) and our own observations we choose a natural/organic visual appearance and behavior. However, the first design with the two ellipses felt not right, since all movements were in reaction to an approaching finger and when the finger didn't move the target did not move. Therefore we added additional ellipses, which moved independently (in random directions) within a bounding range in the background. We added this ambiguous or autonomous design element into the proxemic touch target design to improve the lifelike character (and perceived degree of agency) of the touch target. In order to improve the perceived aesthetic quality of the proxemic touch target we explored color-change behaviors (see Figure 4a).

Instead of shrinking the whole target for a change-size behavior, we chose to increase the color transparency towards the outer border of the touch target. Doing this resulted in a smoother visual experience of the target shrinking and vanishing to the background.

\subsection{User Study}

In order to explore the second research question by testing if the proxemic touch target design would be perceived indeed as attractive and desirable, we conducted a between subjects study comparing the proxemic touch target design against two control designs (i.e, static condition, and animated non-proxemic condition). We chose to use two control groups, since in addition to comparing the proxemic version against the base line (i.e., static version) we needed to account for any effects of the background animation, which was not part of the proxemic behavior.

In sum, the independent variable of our study consisted of three levels: (i) proxemic, (ii) animated, and (iii) static. Considering size and color, we choose for the static and animated versions the visual appearance of the proxemic version when a finger is right about to touch the target (e.g., targets presented at right hand side of Figure $4 \mathrm{c}$ and $\mathrm{d}$ ). Our concrete assumption was that the proxemic condition will be perceived best considering perceived functional and perceived aesthetic qualities. In order to address both kinds of qualities we chose to use the attrakDiff questionnaire, which measures pragmatic quality (PQ), hedonic quality (a combination of the measures HQS and HQI), and overall attractiveness (ATT). The HQS measure is related to a product's perceived capability to satisfy a person's desire of self-improvement, the HQI measure is related to a product's perceived capability to communicate others a valuable identity, and ATT measures general/global attractiveness of a product [15]. We chose to use the attrakDiff, since in addition 
orient and expand towards index finger

a)

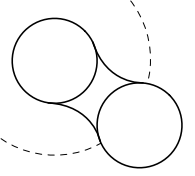

add functional behavior add background animation

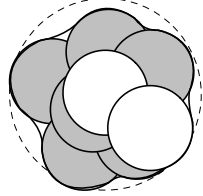

add ambiguous movement, allow users to interpret movement more personally

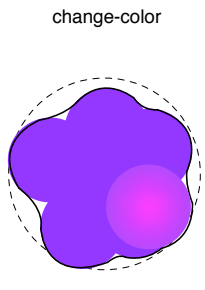

add "aesthetic" qualties

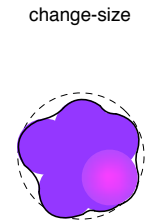

b)
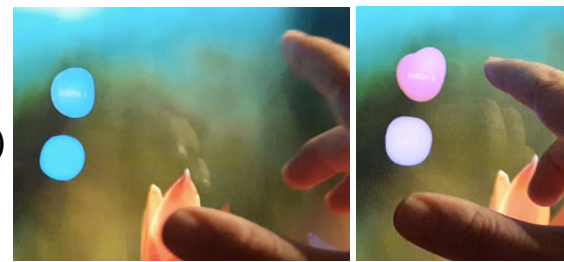

screenshots with two proxemic touch targets on the screen

ow design to disappea

C)

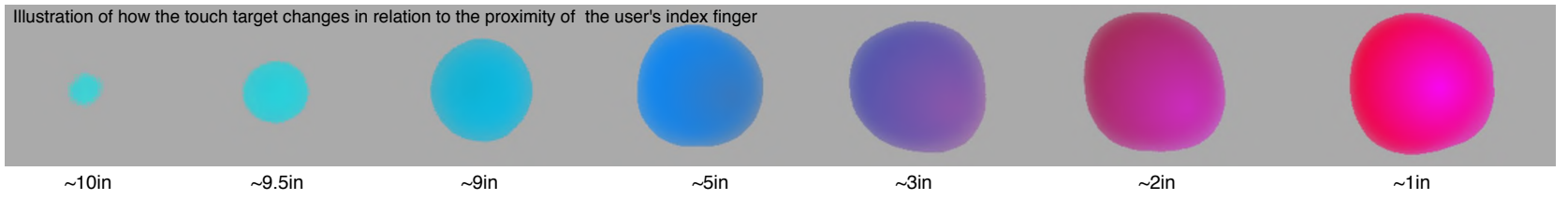
to the background
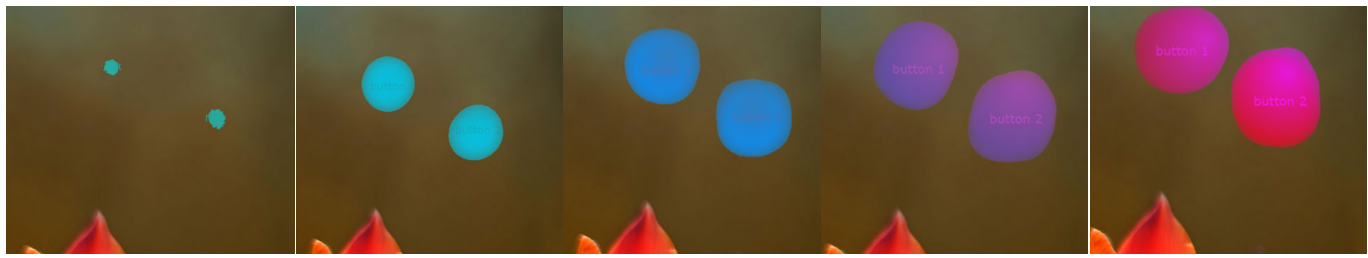

Examples with multiple targets, background images and target labels
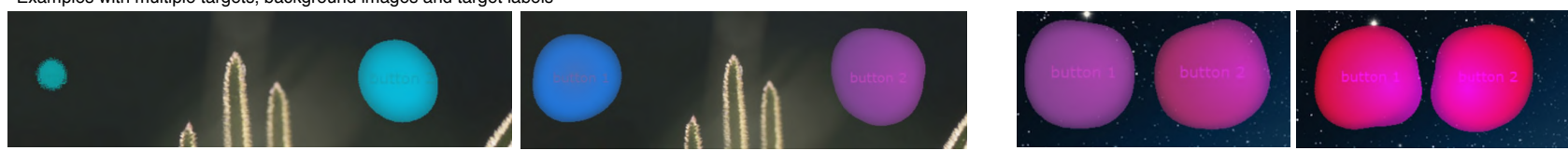

Figure 4: a) Overview of the design decisions towards an iterated proxemic touch target design. b) Photos of a user interacting with the proxemic touch targets. c) Screenshots of how targets change in relation to an approaching finger. d) Screenshots with multiple targets illustrating how targets adapt in relation to an approaching finger.

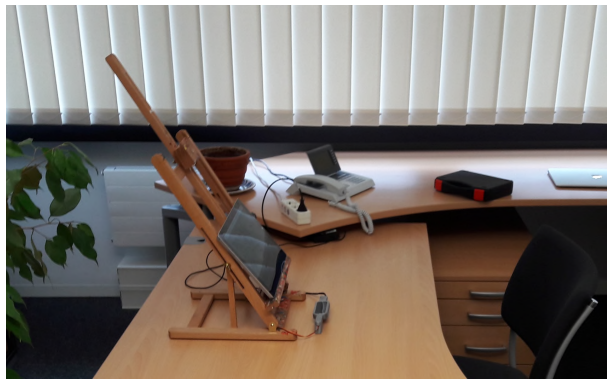

Figure 5: Setup and apparatus for the second user study.

to measuring perceived PQ (i.e., traditional usability) it provides with HQS, HQI and ATT a finer discrimination of what we have so far simply referred to as aesthetic qualities.

\subsection{Participants and Apparatus}

For our study 36 participants (4f, 32m, between 21-34 years of age) (who reported not to have any color weakness and used a device with a touch screen on a daily basis) were recruited at the university campus and were either students or university staff. Instead of using an iPad for this study a Microsoft Surface Pro 2 device was used. We did this since the Surface device provided a USB connection for the Leap Motion controller, improving the overall performance and limiting any delay due to the wireless connection. Figure 5 presents the final physical setup. The study was conducted in an empty office setting and 12 participants tested each condition.

\subsection{Procedure}

We informed participants that the goal of the study was to evaluate the design of a general purpose (e.g., buttons on home screens of Smartphones) touch target design and that we have prepared a simple application that provides them different views and an impression of the touch target design in different contexts.

In each condition participants were instructed to touch any of the touch targets in order to navigate to the next view. We also instructed them that in different views touch target positions and background images would be different to provide them a range of different scenarios. In each condition we used the same application consisting of 20 views. By touching a touch target users could navigate to the next view. In the first ten views one touch target was positioned randomly on the screen (background was kept gray). In the subsequent views pre-defined typical wallpaper images were shown. In six of the views two targets were positioned randomly 


\begin{tabular}{l}
\hline technical - human \\
\hline complicated - simple \\
\hline impractical - practical
\end{tabular}

Figure 6: Overview of mean ratings for each item of the attrakDiff questionnaire. The items are sorted from left ot right (negative to positive) and from top to bottom with the top 7 items measuring $P Q$, followed by the 7 items measuring $H Q I$, then the 7 items measuring $H Q S$, and last but not least the 7 items measuring ATT.

and in four of the views targets were positioned in fixed positions (i.e., horizontally next to each other, horizontally apart from each other, vertically next to each other, and vertically apart from each other). We did this since touch targets are typically positioned next to each other and horizontally or vertically. Participants could take as much time as they needed in each condition and also repeat the views for as long as they needed. Afterwards they were asked to rate the design using the attrakDiff questionnaire. A single session took about 15 minutes.

\subsection{Data Analysis and Results}

In the following we present the analysis of the "quantitative" data collected in the user study using the attrakDiff questionnaire. First, general trends based on graphical illustrations of the data are presented. Then, results will be interpreted based on fitting a statistical model to the data.

5.4.1 General Trends. Figure 6 presents an overview of how each item of the attrakDiff was rated in mean in all three conditions. In most items the proxemic design was rated better than the static and the animated design. An obvious exception is the item "undemanding-challenging" which contributes to the measure HQS. Figure 7 provides an overview of mean values for each of the independent variables (i.e., PQ, HQS, HQI, ATT) measured each by 7 items of the questionnaire. It seems that the proxemic design is rated positive on all measurements with the highest difference in measurement ATT. Furthermore, the static design is rated worst, considering all outcome measures but HQS.

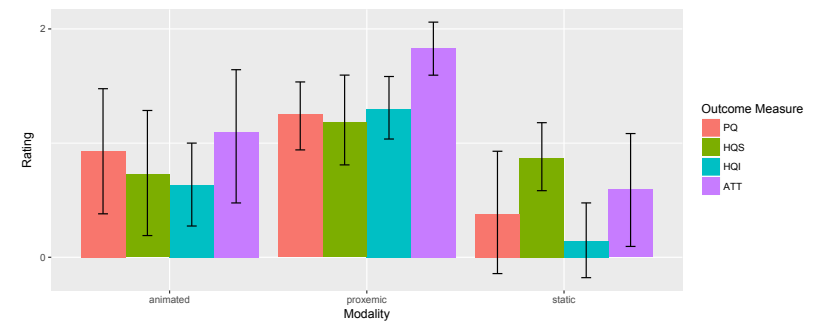

Figure 7: Overview of mean values for each condition and dependent variable (i.e., PQ, HQS, HQI, and ATT) measured by the attrakDiff questionnaire. Error bars denote confidence intervals.

5.4.2 Statistical Analysis. We used a multiple analysis of variance (MANOVA) with between subjects measurements in order to test the difference between the different touch target designs (i.e., static, animated, and proxemic) across the dependent variables measured by the attrakDiff questionnaire (i.e., PQ, HQS, HQI, and ATT ). Using Pillai's trace, there was a significant effect of touch target design on PQ, HQS, HQI and ATT, V = 0.46, $F(8,62)=2.34, p<.05$. Separate univariate ANOVAs on the variables PQ, HQS, HQI, and ATT revealed significant design effects on $\mathrm{HQI} F(2,33)=11.73, p<.001$, and ATT $F(2,33)=6.23, p<.01$. However, non-significant treatment effects were revealed for PQ, $F(2,33)=2.97, p=.065$, and HQS, $F(2,33)=.98, p=.38$.

Since, we hypothesized that the proxemic design would improve pragmatic and hedonic qualities we conducted planned contrasts, which revealed that the proxemic design significantly increased ATT compared to the static design, $t(33)=-3.5, p<$ .01 (one-tailed), and significantly increased ATT compared to the animated design, $t(33)=-2.09, p<.05$ (one-tailed). Furthermore, the proxemic design significantly increased $\mathrm{PQ}$ compared to the static design, $t(33)=-2.41, p<.05$ (one-tailed). However, the comparison to the animated design on $\mathrm{PQ}$ was non-significant, $t(33)=-.08, p=.38$ (one-tailed). The difference between the proxemic design on HQS compared to the static design, $t(33)=$ $-.94, p=.35$ (one-tailed), and compared the animated design was non-significant, $t(33)=-1.38, p=.18$ (one-tailed).

The proxemic design increased significantly on HQI compared to the static design $t(33)=-4.82, p<.05$ (one-tailed). However, the comparison to the animated design was non-significant, $t(33)=$ $-2.79, p<.05$ (one-tailed).

As expected the difference between the animated and static design was less on all dependent variables. The only significant difference between the animated and the static design was on HQI $t(33)=-2.04, p=.049$ (one-tailed). 
Overall the statistical analysis ensured that the proxemic design was perceived significantly more attractive than both the static and animated designs. While the proxemic design was also perceived significantly more pragmatic compared to the static design, the difference between the proxemic and animated design was not perceived as significant. Some of the comments made by the participants considering the animated and static design may explain this result. One participant in the static group argued that when background images are displayed it is sometimes harder to spot the touch target, because the participant was used to interaction with perfect spheres or rectangles. Another participant in the animated group highlighted that animating the touch target actually helped to spot them on the screen.

While we assumed, that the background movement would improve perceived aesthetic qualities, it seems that in the specific application context of navigating through views when targets are positioned on different (random) locations on the screen with varying images as backgrounds the animation is associated with a pragmatic quality, because it helps users spot the touch target. When we check the confidence intervals in Figure 7 for PQ and the animated design, we can see that the participants' perceptions varied more then in the proxemic design. Considering the hedonic qualities (HQS and HQI) the results highlight that the proxemic design seems only to influence HQI. The HQI measure is related to a design's perceived capability to communicate others a valuable identity, in contrast to HQS explains a design's perceived capability to satisfy a person's desire of self-improvement.

\section{CONCLUSION AND FUTURE WORK}

We have argued that proxemic touch targets have the potential to establish physical/bodily and emotional connections through a sensory-motoric coupling between the aimed movement in mid-air of the user and the movement-based feedback of targets. Because the same sensory-motoric connection is missing in state of the art static touch targets, which register users' interactions only on the physical screen and for the time period of the physical touch, we have hypothesized that proxemic touch target designs can result in enhanced experiential qualities associated with touch-screen interaction. We have presented two studies, which explored two research questions, considering the UX of proxemic touch targets.

First, we reported on a qualitative study with five experts, which aimed to improve our understanding of how various visual features may impact user experience. We have learned, for example that changing color, orientation, and form of targets and how targets felt alive was perceived as very intriguing and was preferred by experts, while changing size and position was perceived mainly as useful and functional (on a subjective and experiential level).

Previous work (e.g., $[1,2,29]$ ) has demonstrated that expanding and moving targets improves target acquisition performance. The observations we made in the first study suggested that changing target color, orientation, and form would mainly influence hedonic qualities. Thus, we hypothesized that combining change-types that were described by the experts as "functional" and "aesthetic" would improve both qualities and consequently result in desirable proxemic target designs.
We tested this hypothesis in a second study comparing an exemplary proxemic design with non-proxemic versions of the same touch target design. As assumed, the results of the second study with 36 participants have shown positive effects of proxemic touch targets on pragmatic and hedonic qualities. Applying the attrakDiff as a measurement tool of user experience provided in-depth insights (e.g., see Figure 6 presents all 28 items) by discriminating the aesthetic qualities associated with the proxemic touch target design further into hedonic qualities (i.e., HQI and HQS) and general attractiveness (i.e., ATT). The second study mainly establishes the attractiveness of proxemic touch targets that not simply expand but "behave" in a reactive and as some experts and users considered in a "life-like" manner by changing, color, form, etc.

We think that the implicit synchrony between hand/finger movement and how targets' change reinforced experts'/users' "social connection" to targets, producing a resonating interaction form, which neither previous work nor we explicitly addressed. But which is something that needs more focus in our future work. By establishing the attractiveness of proxemic touch targets we hope to have closed a research gap towards exploring the various aspects of user experience of proxemic touch targets and how they can transform future touch-based interfaces.

Since, proxemic targets have the ability to adapt they can be used in a broad range of applications, addressing potential contextual constraints. We have already mentioned as exemplary application contexts driver interfaces, retail applications, and multi-user settings in general. We believe that tourist guide applications (e.g., [4]), which offer multimodal and multilingual service would also benefit from proxemic targets. Based on our inquiries, we have learned that combining touch and mid-air input as modalities not only has potential to improve performance and productivity (as suggested by several researchers in related work), but that the combination opens up a valuable and contrasting design space to address other interaction qualities, including desirable interaction designs. In this paper, we have put less focus on limits of the interaction technique, such as potential issues when many touch targets are cluttered together [1]. Depending on the specific application such limits can also influence users' experiences. In our experience, designing proxemic targets is not a straight forward task but requires carefully conducted user tests.

In practice, we believe that proxemic touch target designs could especially help novice users and during "slow" interaction settings. While pre-touch proxemics may have little benefits for a proficient user typing rapidly on a virtual keyboard of a mobile device, for a novice user of, for example a smart watch it could help discover the affordances offered by the watche's touch sensitive display in a beautiful and useful manner. One could also consider pre-touch proxemics for an alternative way to implement gesture teaching mechanism [18] or to help users to deal with (or understand) affordances of interfaces designed in foreign or multiple languages.

In a world with increasing complexity of interaction opportunities pre-touch proxemics may serve a greater purpose in future by helping discover interaction possibilities with touch enabled devices. We will dedicate future work to explore how touch targets can communicate their affordances through proxemic behaviors in both functional and attractive ways. 


\section{REFERENCES}

[1] Ilhan Aslan, Alina Krischkowsky, Alexander Meschtscherjakov, Martin Wuchse, and Manfred Tscheligi. 2015. A Leap for Touch: Proximity Sensitive Touch Targets in Cars. In Proceedings of the 7th International Conference on Automotive User Interfaces and Interactive Vehicular Applications (AutomotiveUI '15). ACM, New York, NY, USA, 39-46. https://doi.org/10.1145/2799250.2799273

[2] Ilhan Aslan, Thomas Meneweger, Verena Fuchsberger, and Manfred Tscheligi 2015. Sharing Touch Interfaces: Proximity-Sensitive Touch Targets for TabletMediated Collaboration. In Proceedings of the 2015 ACM on International Conference on Multimodal Interaction (ICMI '15). ACM, New York, NY, USA, 279-286. https://doi.org/10.1145/2818346.2820740

[3] Ilhan Aslan, Andreas Uhl, Alexander Meschtscherjakov, and Manfred Tscheligi 2014. Mid-air Authentication Gestures: An Exploration of Authentication Based on Palm and Finger Motions. In Proceedings of the 16th International Conference on Multimodal Interaction (ICMI '14). ACM, New York, NY, USA, 311-318. https: //doi.org/10.1145/2663204.2663246

[4] Ilhan Aslan, Feiyu Xu, Hans Uszkoreit, Antonio Krüger, and Jörg Steffen. 2005 COMPASS2008: Multimodal, multilingual and crosslingual interaction for mobile tourist guide applications. In INTETAIN. Springer, 3-12.

[5] Till Ballendat, Nicolai Marquardt, and Saul Greenberg. 2010. Proxemic Interaction: Designing for a Proximity and Orientation-aware Environment. In ACM International Conference on Interactive Tabletops and Surfaces (ITS '10). ACM, New York, NY, USA, 121-130. https://doi.org/10.1145/1936652.1936676

[6] Virginia Braun and Victoria Clarke. 2006. Using thematic analysis in psychology. Qualitative research in psychology 3, 2 (2006), 77-101.

[7] Xiang 'Anthony' Chen, Julia Schwarz, Chris Harrison, Jennifer Mankoff, and Scott E. Hudson. 2014. Air+Touch: Interweaving Touch and In-air Gestures. In Proceedings of the 27th Annual ACM Symposium on User Interface Software and Technology (UIST '14). ACM, New York, NY, USA, 519-525. https://doi.org/10. $1145 / 2642918.2647392$

[8] Karl Anders Ericsson and Herbert Alexander Simon. 1993. Protocol analysis. MIT press Cambridge, MA

[9] P. M. Fitts. 1992. The information capacity of the human motor system in controlling the amplitude of movement. 1954. J Exp Psychol Gen 121, 3 (Sept. 1992), 262-269.

[10] William W. Gaver, Jacob Beaver, and Steve Benford. 2003. Ambiguity As a Resource for Design. In Proceedings of the SIGCHI Conference on Human Factors in Computing Systems (CHI '03). ACM, New York, NY, USA, 233-240. https: //doi.org/10.1145/642611.642653

[11] Antonio Gomes, Andrea Nesbitt, and Roel Vertegaal. 2013. MorePhone: A Study of Actuated Shape Deformations for Flexible Thin-film Smartphone Notifications. In Proceedings of the SIGCHI Conference on Human Factors in Computing Systems (CHI '13). ACM, New York, NY, USA, 583-592. https://doi.org/10.1145/2470654.2470737

[12] Saul Greenberg, Nicolai Marquardt, Till Ballendat, Rob Diaz-Marino, and Miaosen Wang. 2011. Proxemic Interactions: The New Ubicomp? interactions 18,1 (Jan 2011), 42-50. https://doi.org/10.1145/1897239.1897250

[13] Jože Guna, Grega Jakus, Matevž Pogačnik, Sašo Tomažič, and Jaka Sodnik. 2014 An analysis of the precision and reliability of the leap motion sensor and its suitability for static and dynamic tracking. Sensors 14, 2 (2014), 3702-3720.

[14] Edward Twitchell Hall and Edward T Hall. 1969. The hidden dimension. Vol. 1990 Anchor Books New York.

[15] Marc Hassenzahl, Micheal Burmester, Franz Koller, H Brau, S Diefenbach, M Hassenzahl, F Koller, M Peissner, and K Röse. 2008. Der User Experience (UX) auf der Spur: Zum Einsatz von www. attrakdiff. de. Usability Professionals 2008 (2008), 78-82

[16] Fabian Hemmert, Matthias Löwe, Anne Wohlauf, and Gesche Joost. 2013. Animate Mobiles: Proxemically Reactive Posture Actuation As a Means of Relational
Interaction with Mobile Phones. In Proceedings of the 7th International Conference on Tangible, Embedded and Embodied Interaction (TEI '13). ACM, New York, NY, USA, 267-270. https://doi.org/10.1145/2460625.2460669

[17] Ken Hinckley, Seongkook Heo, Michel Pahud, Christian Holz, Hrvoje Benko, Abigail Sellen, Richard Banks, Kenton O'Hara, Gavin Smyth, and William Buxton. 2016. Pre-Touch Sensing for Mobile Interaction. In Proceedings of the $2016 \mathrm{CHI}$ Conference on Human Factors in Computing Systems (CHI '16). ACM, New York, NY, USA, 2869-2881. https://doi.org/10.1145/2858036.2858095

[18] Kay Hofmeester and Jennifer Wolfe. 2012. Self-revealing Gestures: Teaching New Touch Interactions in Windows 8. In CHI'12 Extended Abstracts on Human Factors in Computing Systems (CHI EA '12). ACM, New York, NY, USA, 815-828. https://doi.org/10.1145/2212776.2212855

[19] Talia Konkle, Timothy F Brady, George A Alvarez, and Aude Oliva. 2010. Conceptual distinctiveness supports detailed visual long-term memory for real-world objects. Fournal of Experimental Psychology: General 139, 3 (2010), 558.

[20] Michael McGuffin and Ravin Balakrishnan. 2002. Acquisition of Expanding Targets. In Proceedings of the SIGCHI Conference on Human Factors in Computing Systems (CHI '02). New York, NY, USA, 57-64. https://doi.org/10.1145/503376. 503388

[21] David E Meyer, Richard A Abrams, Sylvan Kornblum, Charles E Wright, and JE Keith Smith. 1988. Optimality in human motor performance: ideal control of rapid aimed movements. Psychological review 95, 3 (1988).

[22] Jörg Müller, Gilles Bailly, Thor Bossuyt, and Niklas Hillgren. 2014. MirrorTouch: Combining Touch and Mid-air Gestures for Public Displays. In Proceedings of the 16th International Conference on Human-computer Interaction with Mobile Devices \&\#38; Services (MobileHCI '14). ACM, New York, NY, USA, 319-328. https: //doi.org/10.1145/2628363.2628379

[23] Amanda Parkes, Ivan Poupyrev, and Hiroshi Ishii. 2008. Designing Kinetic Interactions for Organic User Interfaces. Commun. ACM 51, 6 (June 2008), 58-65. https://doi.org/10.1145/1349026.1349039

[24] Esben W. Pedersen, Sriram Subramanian, and Kasper Hornbæk. 2014. Is My Phone Alive?: A Large-scale Study of Shape Change in Handheld Devices Using Videos. In Proceedings of the 32Nd Annual ACM Conference on Human Factors in Computing Systems (CHI '14). ACM, New York, NY, USA, 2579-2588. https: //doi.org/10.1145/2556288.2557018

[25] Majken K. Rasmussen, Esben W. Pedersen, Marianne G. Petersen, and Kasper Hornbæk. 2012. Shape-changing Interfaces: A Review of the Design Space and Open Research Questions. In Proceedings of the SIGCHI Conference on Human Factors in Computing Systems (CHI '12). ACM, New York, NY, USA, 735-744. https://doi.org/10.1145/2207676.2207781

[26] Anne Roudaut, Abhijit Karnik, Markus Löchtefeld, and Sriram Subramanian. 2013. Morphees: Toward High "Shape Resolution" in Self-actuated Flexible Mobile Devices. In Proceedings of the SIGCHI Conference on Human Factors in Computing Systems (CHI '13). ACM, New York, NY, USA, 593-602. https://doi. org $/ 10.1145 / 2470654.2470738$

[27] Maxine Sheets-Johnstone. 2011. The primacy of movement. Vol. 82. John Benjamins Publishing.

[28] Jonas Togler, Fabian Hemmert, and Reto Wettach. 2009. Living Interfaces: The Thrifty Faucet. In Proceedings of the 3rd International Conference on Tangible and Embedded Interaction (TEI '09). ACM, New York, NY, USA, 43-44. https: //doi.org/10.1145/1517664.1517680

[29] Xing-Dong Yang, Tovi Grossman, Pourang Irani, and George Fitzmaurice. 2011. TouchCuts and TouchZoom: Enhanced Target Selection for Touch Displays Using Finger Proximity Sensing. In Proceedings of the SIGCHI Conference on Human Factors in Computing Systems (CHI '11). ACM, New York, NY, USA, 2585-2594. https://doi.org/10.1145/1978942.1979319 